2015

\title{
Multidimensional Coherent Spectroscopy Of A Semiconductor Microcavity
}

Brian L. Wilmer

Felix Passmann

Michael Gehl

Galina Khitrova

Alan D. Bristow

Follow this and additional works at: https://researchrepository.wvu.edu/faculty_publications

\section{Digital Commons Citation}

Wilmer, Brian L.; Passmann, Felix; Gehl, Michael; Khitrova, Galina; and Bristow, Alan D., "Multidimensional Coherent Spectroscopy Of A Semiconductor Microcavity" (2015). Faculty Scholarship. 603.

https://researchrepository.wvu.edu/faculty_publications/603 


\title{
Multidimensional coherent spectroscopy of a semiconductor microcavity
}

\author{
Brian L. Wilmer, ${ }^{1}$ Felix Passmann, ${ }^{1,2}$ Michael Gehl, ${ }^{3}$ Galina Khitrova, ${ }^{3}$ and Alan D. Bristow ${ }^{1, *}$ \\ ${ }^{1}$ Department of Physics and Astronomy, West Virginia University, Morgantown, West Virginia 26501-6315, USA \\ ${ }^{2}$ Experimentelle Physik 2, TU Dortmund, Dortmund 44221, Germany \\ ${ }^{3}$ College of Optical Sciences, The University of Arizona, Tucson, Arizona 85721-0094, USA \\ (Received 4 March 2015; revised manuscript received 1 May 2015; published 20 May 2015)
}

\begin{abstract}
Rephasing and nonrephasing two-dimensional coherent spectra map the anticrossing associated with normalmode splitting in a semiconductor microcavity. For a $12-\mathrm{meV}$ detuning range near zero detuning, it is observed that there are two diagonal features related to the intra-action of exciton-polariton branches and two off-diagonal features related to coherent interaction between the polaritons. At negative detuning, the line shape properties of the diagonal intra-action features are distinguishable and can be associated with cavitylike and excitonlike modes. A biexcitonic companion feature is observed, shifted from the exciton feature by the biexciton binding energy. Closer to zero detuning, all features are enhanced and the diagonal intra-action features become nearly equal in amplitude and linewidth. At positive detuning the excitonlike and cavitylike characteristics return to the diagonal intra-action features. Off-diagonal interaction features exhibit asymmetry in their amplitudes throughout the detuning range. The amplitudes are strongly modulated (and invert) at small positive detuning, as the lower polariton branch crosses the bound biexciton energy determined from negatively detuned spectra.
\end{abstract}

DOI: 10.1103/PhysRevB.91.201304

PACS number(s): 73.21.Fg, 78.47.J-, 78.47.nj

Semiconductor microcavities supporting excitonpolaritons [1,2] are used in optoelectronic applications [3-5] and provide a platform for exploring exotic coherent physical phenomena [6-14]. The normal-mode coupling between the photonic cavity mode $(\gamma)$ and the exciton resonance $(\mathrm{X})$ enhances both the linear and nonlinear optical interactions [15]. Transient four-wave mixing (FWM) confirms that strong exciton-cavity interactions modify the temporal behavior of the coherent response and many-body Coulomb correlations determine the exact dynamics [16], which affects dissipation $[7,17,18]$ and coherent control [19,20]. Biexciton-polaritons also contribute to the overall emission signal, even though the biexciton binding energy is only slightly altered by the cavity $[21,22]$.

Multidimensional coherent spectroscopy (MDCS) is based on and supersedes FWM. At optical frequencies, MDCS has been utilized to study various semiconductor nanostructures [23-29]. This technique retains both time and frequency resolution, is able to unambiguously distinguish a variety of quantum pathways (including those with nonradiative steps) [30], and can separate homogeneous and inhomogeneous broadening [31]. To date, two-quantum, and higherorder [32], coherent spectra have shown that many-body interactions dominate the signals, including a contribution from bound biexcitons for excitation with the correct polarization configuration. Two-dimensional coherent spectroscopy (2DCS) has also examined coherence and control of excitonic qubits in microcavity pillars [33].

Despite the extensive fundamental and applied studies of microcavity exciton-polaritons, the anticrossing has not been systematically mapped using MDCS. In this paper normal-mode splitting of a semiconductor microcavity and the associated exciton-polariton branches are mapped using rephasing and nonrephasing 2DCS. This study is performed over a range of energy detuning $\left(\Delta=E_{\gamma}-E_{\mathrm{X}}\right)$ near the

\footnotetext{
*alan.bristow@mail.wvu.edu
}

anticrossing, where $E_{\gamma}$ and $E_{\mathrm{X}}$ correspond the cavity-mode and exciton-mode energies, respectively. The detuning dependence of spectral features, related to intra-action (diagonal features) of and interaction (off-diagonal features) between polariton branches, informs us about the coupling between the cavity and excitonic or biexcitonic modes. For example, a contribution from a bound biexciton is isolated at negative detuning, which is convolved with the off-diagonal features at positive detuning and strongly modulates their relative amplitudes.

The experimental setup is described fully elsewhere [34]. In brief, the laser source is a mode-locked Ti:sapphire oscillator that produces $100-\mathrm{fs}$ pulses. A MONSTR is used to create and phase control four identical pulses arranged on the corners of a box. As shown in Fig. 1, three pulses impinge the microcavity sample, which resides in an optical cryostation at the focus and crossing point of the beams. A tracer ( $\mathrm{Tr}$ ) beam is used for alignment and blocked for the FWM and 2DCS measurements. All measurements are performed in the third-order nonlinear optical regime, excited with average powers of $0.2-0.7 \mathrm{~mW}$ per beam. In a third-order perturbation excitation scheme, the signal is generated from interaction by all three excitation pulses. The excitation sequence is shown in the inset of Fig. 1: The first pulse creates a coherent superposition between the ground and excited states, the second pulse then creates a population in either the ground or excited state, and the third pulse converts the population into a radiating polarization. This polarization is emitted as a transient FWM signal, which is collected in transmission mode and directed to a spectrometer and CCD camera.

The microcavity sample (denoted NMC73) was grown by molecular beam epitaxy on a GaAs substrate [1]. The mirrors consist of GaAs/AlAs (14.5 and 12 bilayer) distributed Bragg reflectors separated by a wedged $\lambda$ GaAs cavity, with a cavity mode close to $830 \mathrm{~nm}$. In the center of the cavity, at the antinode of its electric field, is a single 8-nm $\mathrm{In}_{0.04} \mathrm{Ga}_{0.96}$ As quantum well. The reflection properties at low temperature (not shown) exhibit the typical normal-mode splitting expected for such a 


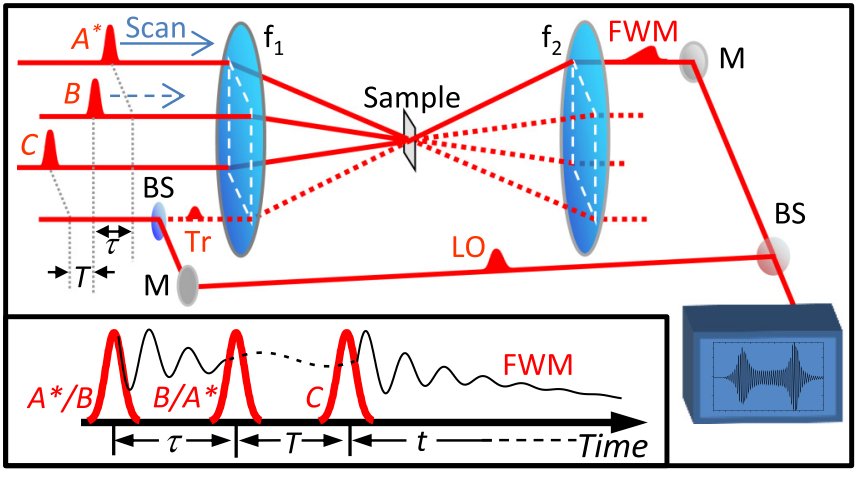

FIG. 1. (Color online) Experimental setup for the multidimensional coherent spectroscopy ( $\mathrm{f}=$ lens, $\mathrm{M}=$ mirror, $\mathrm{BS}=$ beam splitter, FWM $=$ four-wave-mixing signal, $\tau=$ period between pulses $\mathrm{A}$ and $\mathrm{B}, T=$ period between pulses $\mathrm{B}$ and $\mathrm{C}, t=$ period after pulse $\mathrm{C}$ triggers the emission, $\mathrm{Tr}=$ tracer beam, traces the phase-matched direction, and $\mathrm{LO}=$ local oscillator for spectral interferometry). The inset shows the excitation pulse sequences.

structure. Translating the sample detunes the cavity mode with respect to the bare exciton energy.

Spectrally resolved FWM is acquired as a precursor to performing 2DCS with $\tau=0 \mathrm{fs}$ and $T=100 \mathrm{fs}$. The inset of Fig. 2 shows a typical spectrum, revealing resonances associated with the lower (LP) and upper (UP) polariton branches. Figure 2 shows the spectral positions extracted from spectra measured at different positions on the sample. The detuning $\Delta$ is determined for each spectrum by modeling the data using

$$
E_{\mathrm{UP} / \mathrm{LP}}(\Delta)=1 / 2\left[2 E_{\mathrm{X}}+\Delta \pm\left(\Delta^{2}+E_{\mathrm{VRS}}^{2}\right)^{1 / 2}\right]
$$

where $E_{\mathrm{VRS}}$ is the vacuum Rabi splitting, a measure of the coupling between the optical field and the excitons. It is found that $E_{\mathrm{VRS}}=3.1 \pm 0.1 \mathrm{meV}$ and $E_{\mathrm{X}}=1491.3 \pm 0.03 \mathrm{meV}$, which both agree well with results on similar structures [1]. The inset spectrum is then indicated as a dashed vertical line at $\Delta \simeq-3.75 \mathrm{meV}$. Microcavities are sensitive to the angle of incidence, since the dispersion varies with the in-plane wave vector. Here the external angle of incidence for each beam is approximately $7^{\circ}$ and the axis of the box of pulses is at normal incidence. Each beam couples to slightly different polariton states in momentum space, which may lift the measured energy and lower the overall signal strength. However, close to zero detuning, the FWM emission requires attenuation of at least ND2.5, indicating minimal momentum-related signal reduction and excitation within the parabolic region of the in-plane momentum [35].

For 2DCS measurements, the transient FWM is collected in the spectrometer along with a phase-stabilized local oscillator (LO) pulse, such that complex spectra can be recorded by spectral interferometry. Spectra are acquired for a range of time delays $\tau$ scanned in phase-stabilized increments. A numerical Fourier transform is performed to convert $\tau$ to $\omega_{\tau}$. If the conjugate pulse $A^{*}$ is scanned backward in time (toward the sample), the time-ordering results in the phase-matching condition $k_{s}=-k_{A^{*}}+k_{B}+k_{C}$. Transient FWM exhibits a photon echo for inhomogeneously broadened systems. This method records a rephasing $2 \mathrm{D}$ spectrum $S_{I}\left(-\omega_{\tau}, T, \omega_{t}\right)$,

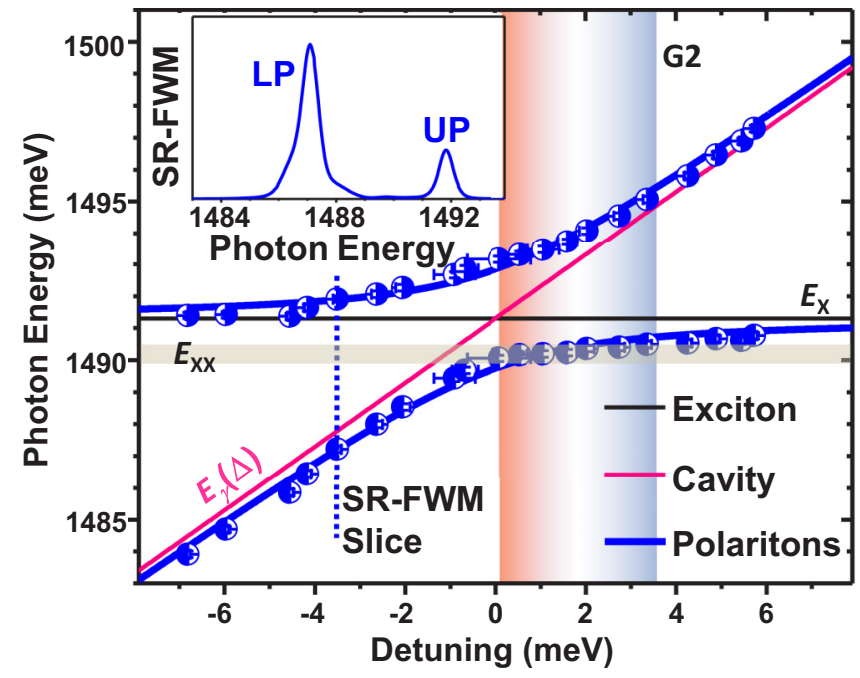

FIG. 2. (Color online) Detuning dependence of the features observed in spectrally resolved four-wave mixing (FWM). A typical FWM spectrum is shown in the inset, from which the center energies of the lower (LP) and upper (UP) polaritons are extracted for the body of the figure. Solid lines model the vacuum Rabi splitting, the bare exciton energy, and the detuning for each spectrum. Also shown are the expected energy of the bound biexciton $E_{\mathrm{XX}}$ and the detuning range of the G2 profile discussed in Fig. 4.

wherein the diagonal $\left(\hbar \omega_{\tau}=\hbar \omega_{t}\right)$ of the plot is towards the lower right corner, due to the numerical choice of the emission photon energy. Rephasing spectra allow for the separation of homogeneous and inhomogeneous linewidths. Alternatively, if pulse $B$ is scanned instead of pulse $A^{*}$, then the phase matching becomes $k_{s}=k_{B}-k_{A^{*}}+k_{C}$ and the spectrum is nonrephasing, $S_{I I}\left(\omega_{\tau}, T, \omega_{t}\right)$.

Figure 3 shows the absolute field amplitude of the rephasing (bottom row) and nonrephasing (top row) 2DCS results for a range of detuning values from (a) $\Delta=-5 \mathrm{meV}$ to (e) $+4.5 \mathrm{meV}$. Excitation is performed with a mixing time $T=100 \mathrm{fs}$ and collinear polarization (XXXX), where the notation corresponds to the polarization state of the three pump pulses and the emission. Each panel is normalized to the strongest peak for presentation. At each value of $\Delta$ the laser spectrum is overlaid with the nonrephasing spectrum, illustrating that the two resonances are excited equally in each case. This is important for careful comparison of the relative amplitudes of each feature as a function of detuning. Due to the transmission geometry, strong absorption of the tracer beam prevents experimental determination of the global phase using all-optical methods or via spectrally resolved transient absorption [36]. Hence, only amplitude spectra are shown.

In Fig. 3(a) the $\gamma$-like mode is the low-energy feature, denoted A, and is broader than the higher-energy X-like mode, denoted B. From analysis of the line shapes the cross-diagonal width is dominated by the homogeneous linewidths [37] of intra-action features $\mathrm{A}$ and $\mathrm{B}$, yielding values of $\gamma_{\mathrm{LP}}=$ $0.35 \mathrm{meV}$ and $\gamma_{\mathrm{UP}}=0.14 \mathrm{meV}$, respectively. In comparison, the diagonal linewidths, which can be dominated by inhomogeneous broadening, are only slightly wider in each case: $\sigma_{\mathrm{LP}}=0.38 \mathrm{meV}$ and $\sigma_{\mathrm{UP}}=0.17 \mathrm{meV}$. In addition to the diagonal intra-action features, two off-diagonal interaction 


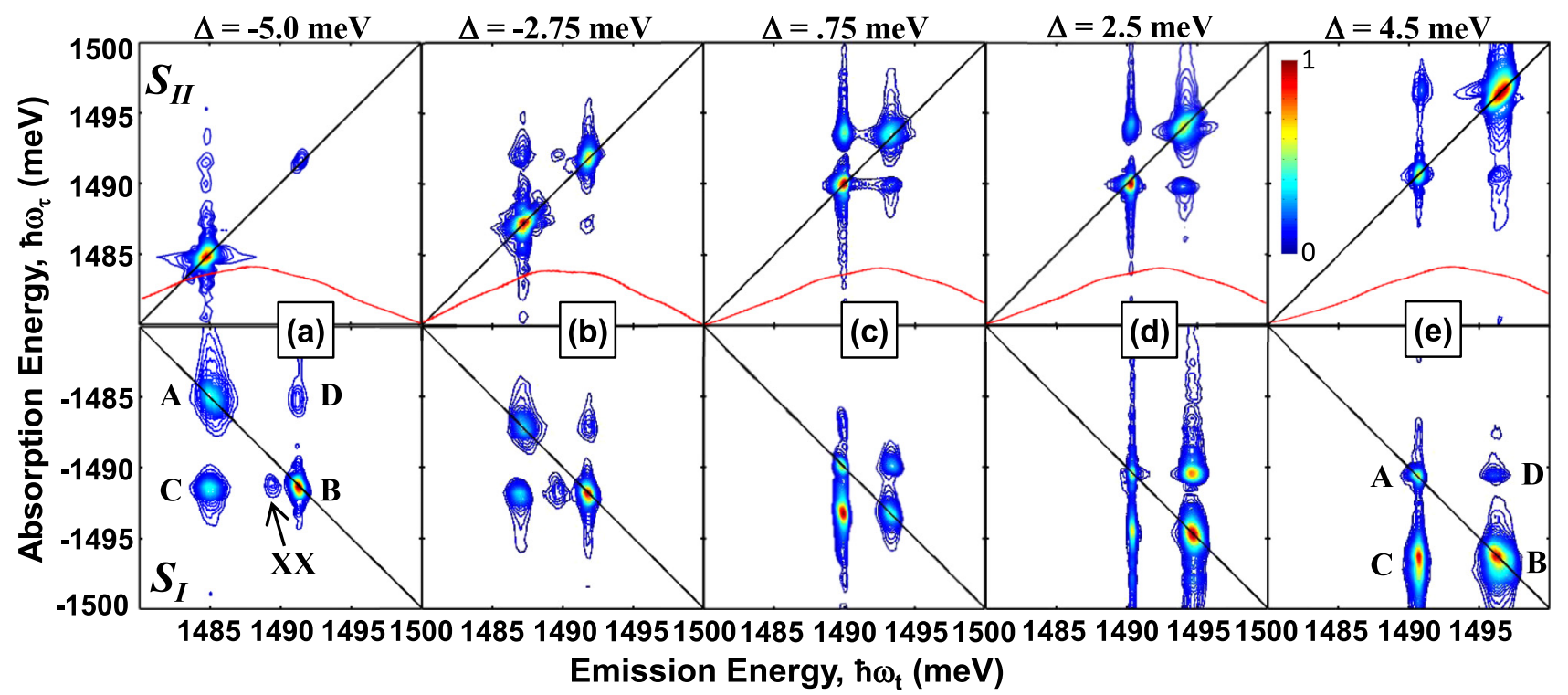

FIG. 3. (Color online) Rephasing $S_{I}$ (bottom row) and nonrephasing $S_{I I}$ (top row) two-dimensional coherent spectra for the labeled range of detuning. Also shown is the laser excitation spectrum for each excitation position. Diagonal intra-action features are A and B, and off-diagonal interaction features are $\mathrm{C}$ and $\mathrm{D}$. Negative detuning also shows a biexciton feature $\mathrm{XX}$.

features $\mathrm{C}$ and $\mathrm{D}$ are observed, which are due to coherent coupling between the A and B features [23].

Increasing $\Delta$ shifts all spectral features toward higher energy. The separation between the A and B modes (projected onto the emission axis) is $\sim 7 \mathrm{meV}$ in (a), decreases as $\Delta$ tends to zero, becoming $3.4 \mathrm{meV}$ in (c), and increases again to $5.6 \mathrm{meV}$ in (e). Close to zero detuning $(\Delta=0.75 \mathrm{meV})$, the homogeneous linewidths of A and B in the $S_{I}$ spectra are $\gamma_{\mathrm{LP}}=$ $0.12 \mathrm{meV}$ and $\gamma_{\mathrm{UP}}=0.18 \mathrm{meV}$, respectively. The properties are nearly identical and $\gamma$-like and X-like characteristics are no longer distinguishable, since it is expected that the observed linewidths should become identical [38]. For larger positive detuning the homogeneous linewidths of $\mathrm{A}$ and $\mathrm{B}$ are $\gamma_{\mathrm{LP}}=0.14 \mathrm{meV}$ and $\gamma_{\mathrm{UP}}=0.24 \mathrm{meV}$, respectively. The $\gamma$-like and $\mathrm{X}$-like characteristics are once again distinguishable, but are not quite the same as for negative detuning. As expected from analysis of the Hopfield coefficients [21], the modes switch and the $\gamma$-like mode is now the upper polariton B. The mode switch is consistent with results where the mirror reflectivity results in a narrower cavity than bare exciton linewidth [38].

Collinear polarization in 2DCS allows for excitation to the biexciton (XX) states [39]. For bound biexcitons, binding energy acts to shift the XX feature laterally from the X-like mode in the emission energy $\hbar \omega_{t}$. This feature is only observed for negative detuning, from which the XX binding energy is determined to be $\sim 1.88 \mathrm{meV}$. The biexciton follows the expected excitation-density dependence and is suppressed for co-circular polarization (data not shown). Its cross-diagonal linewidth is almost identical to that for the X-like mode, which is expected because the quantum pathway that creates the XX feature is a two-step excitation via the exciton. Hence, the linewidth projected on the absorption energy $-\hbar \omega_{\tau}$ should be identical to that for the exciton. The linewidth projection onto $\hbar \omega_{t}$ may be a little wider (tilting the feature away from the diagonal), depending on the degree of correlation of the exciton and biexciton states [40,41]. In this case, the exciton and its biexciton are parallel and are highly correlated.

Nonrephasing $S_{I I}$ are presented for comparison, showing very similar results to $S_{I}$ spectra across the entire detuning range. $S_{I I}$ spectra typically have slightly weaker off-diagonal features, as is observed here. Otherwise, the two diagonal intraaction, two off-diagonal interaction, and biexciton features are all observed as discussed above.

Figure 4 shows the integrated amplitude versus detuning for the four main polaritonic features A through D. In each case, a small area around each feature is integrated. Figure 4(a) shows the total integrated amplitude for a $12-\mathrm{meV}$ detuning range and is normalized to the highest emission strength at $\Delta=0 \mathrm{meV}$. Significant enhancement of the exciton-polariton transition are observed due to normal-mode coupling [42], as the integrated amplitude increases and peak close to zero detuning. The peak shape of extracted total amplitude is asymmetric in detuning [43] and can be fit with two Gaussian line shapes: $\mathrm{G} 1$ is centered at zero detuning and G2 is centered at $1.88 \mathrm{meV}$, corresponding to the biexciton binding energy determined from negatively detuned spectra. Fitted full widths at half maximum of $\mathrm{G} 1$ and $\mathrm{G} 2$ are $\sim 3.39$ and $\sim 3.65 \mathrm{meV}$, respectively. The sum of $\mathrm{G} 1$ and $\mathrm{G} 2$ well represent the $\Delta$-dependence peak shape.

Figures 4(b) and 4(c) show $\Delta$ dependence of the relative amplitude for the individual diagonal intra-action and offdiagonal interaction spectral features. From each spectrum the individual features' integrated amplitudes are extracted and normalized to the total integrated amplitude. At zero detuning the relative amplitudes of the diagonal intra-action features are identical, showing that normal-mode coupling leads to enhancement and equalization of the UP and LP branches. Away from zero detuning the higher-energy diagonal feature $\mathrm{B}$ is always stronger and the overall amplitude of the diagonal features drop at small positive detuning. In contrast, the relative integrated amplitude of the off-diagonal features is smaller at 


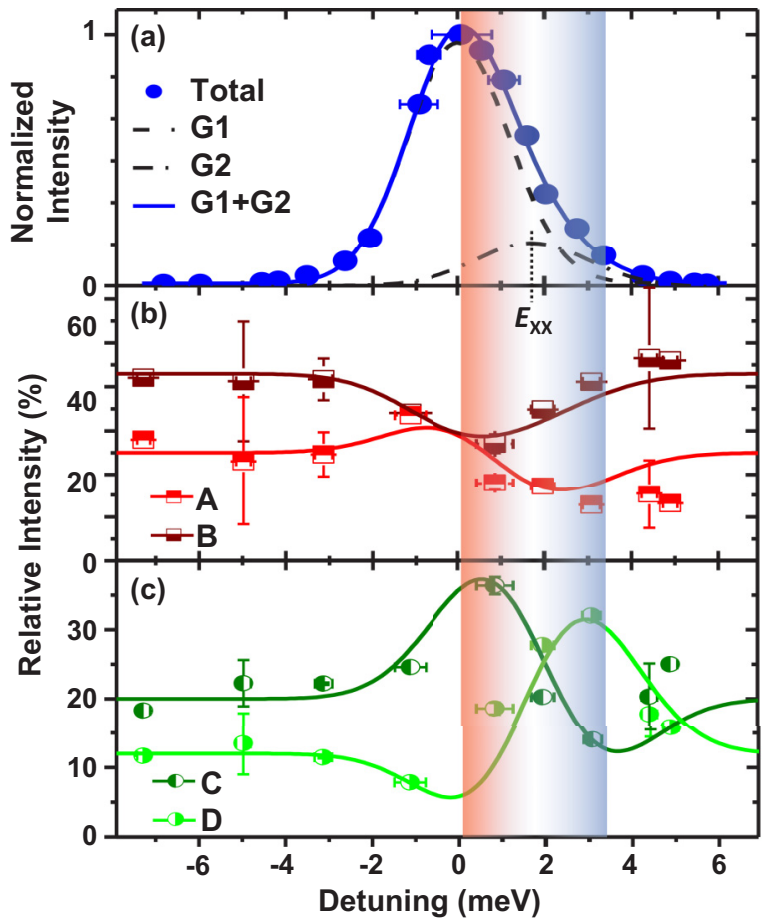

FIG. 4. (Color online) Detuning dependence of the features in the rephasing 2DCS. (a) Shows the normalized amplitude of the entire spectrum, with best fits based on two Gaussian profiles G1 and G2. (b) Shows the relative amplitude of the diagonal features A and B. (c) Shows the relative amplitude of the off-diagonal features $\mathrm{C}$ and D. The solid lines in (b) and (c) model the affect of the two resonances parametrized by the G1 and G2 Gaussian profile in (a).

negative detuning and oscillates for positive detuning. The oscillation position is consistent with the position of the small G2 peak from Fig. 4(a). In this range, the amplitude of peak C increases rapidly, corresponding to the increasing slope of G2, and decreases rapidly to become smaller than $\mathrm{D}$, corresponding to the decreasing slope of G2. Both the width of G2 and the range of $\Delta$ where the oscillations occur agree well with the width of the LP branch passing through the bound biexciton, see Fig. 2.

The solid lines in Figs. 4(b) and 4(c) model the relative amplitudes of the diagonal and off-diagonal features based on the following parameters. First, amplitude offsets for each feature are selected from negatively detuned spectra, yielding $25 \%, 43 \%, 10 \%$, and $12 \%$, for A, B, C, and D, respectively. Second, near $\Delta=0 \mathrm{meV}$, the strength of the two diagonal features equalizes, bringing the two modes close in amplitude in the range of the $\mathrm{G} 1$ profile. This $\sim 9 \%$ deviation is symmetric for each feature. Third, in the region of the $\mathrm{G} 2$ profile $\sim 11 \%$ of the spectral weight is transferred from both diagonal features to the off-diagonal features, most likely due to additional many-particle interaction terms in the quantum pathways to the biexciton manifold. Fourth, the off-diagonal features experience a $\pm d(\mathrm{G} 2) / d \Delta$ modulation in their spectral weight by approximately $\pm 14 \%$, which is most likely due to attraction or repulsion of LP branch as it passes through XX. The latter two effects are complementary evidence of the recently observed Feshbach resonance [14]. Feshbach resonances occur when the energy of two free, yet interacting, polaritons is in resonance with the bound molecular excitonic state. Off-diagonal features are the interaction between the LP and UP branches, so that even though the LP branch alone overlaps with the XX, both off-diagonal amplitudes are modulated and invert. This result arises from coherent coupling by quantum interference of the polaritons through the shared ground state or by a Raman-like coherence between the excited polaritons [30]. 2DCS sensitivity is revealed, because no splitting is observed associated with the Feshbach resonance in the linear spectra, yet the influence of this LP-XX crossing is clear.

In summary, this study has mapped the detuning dependence of the cavity mode through the exciton and biexciton modes of a single quantum well and isolated the coherent response using two-dimensional coherent spectroscopy. Enhancement of the four-wave mixing emission was observed near zero detuning, along with anticrossing of the upper and lower polariton branches. Homogeneous and inhomogeneous linewidths are consistent with those for a wider cavity mode than the bare exciton mode. Amplitudes of the spectral features are highly sensitive to the interaction between exciton, biexciton, and cavity modes, revealing strong modification as bands intersect. This work paves the way for determining contributions through polarization- and excitation-dependent studies using 2DCS and begs full microscopic theoretical treatment to reproduce spectral features. Moreover, these methods can be used to disentangle the coherent and transient phenomena that parallel processes identified in ultracold atomic physics, such as condensation and superfluidity.

The authors wish to acknowledge Steven Cundiff for useful discussions. The work at WVU was supported by the National Science Foundation (CBET-1233795) and the WV Higher Education Policy Commission (HEPC.dsr.12.29). Work at Arizona was supported by AFOSR (FA9550-13-1-0003), NSF-AMOP, and NSF REC-CIAN.
[1] G. Khitrova, H. M. Gibbs, F. Jahnke, M. Kira, and S. W. Koch, Nonlinear optics of normal-mode-coupling semiconductor microcavities, Rev. Mod. Phys. 71, 1591 (1999).

[2] H. M. Gibbs, G. Khitrova, and S. W. Koch, Exciton-polaritons light-semiconductor coupling effects, Nat. Photon. 5, 273 (2011).

[3] S. Christopoulos, G. Baldassarri Hoger von Högersthal, A. J. D. Grundy, P. G. Lagoudakis, A. V. Kavokin, J. J. Baumberg, G. Christmann, R. Butté, E. Feltin, J.-F. Carlin, and
N. Grandjean, Room-temperature polariton lasing in semiconductor microcavities, Phys. Rev. Lett. 98, 126405 (2007).

[4] P. Bhattacharya, T. Frost, S. Deshpande, M. Z. Baten, A. Hazari, and A. Das, Room temperature electrically injected polariton laser, Phys. Rev. Lett. 112, 236802 (2014).

[5] A. Imamoğlu, R. J. Ram, S. Pau, and Y. Yamamoto, Nonequilibrium condensates and lasers without inversion: Exciton-polariton lasers, Phys. Rev. A 53, 4250 (1996). 
[6] H. Deng, G. Weihs, C. Santori, J. Bloch, and Y. Yamamoto, Condensation of semiconductor microcavity exciton polaritons, Science 298, 199 (2002).

[7] J. Kasprzak, M. Richard, S. Kundermann, A. Baas, P. Jeambrun, J. M. J. Keeling, F. M. Marchetti, M. H. Szymańska, R. André, J. L. Staehli, V. Savona, P. B. Littlewood, B. Deveaud, and L. S. Dang, Bose-Einstein condensation of exciton polaritons, Nature (London) 443, 409 (2006).

[8] I. A. Shelykh, Y. G. Rubo, G. Malpuech, D. D. Solnyshkov, and A. Kavokin, Polarization and propagation of polariton condensates, Phys. Rev. Lett. 97, 066402 (2006).

[9] R. Balili, V. Hartwell, D. Snoke, L. Pfeiffer, and K. West, Bose-Einstein condensation of microcavity polaritons in a trap, Science 316, 1007 (2007).

[10] G. Roumpos, M. D. Fraser, A. Löffler, S. Höfling, A. Forchel, and Y. Yamamoto, Single vortex-antivortex pair in an excitonpolariton condensate, Nat. Phys. 7, 129 (2011).

[11] A. Amo, J. Lefrère, S. Pigeon, C. Adrados, C. Ciuti, I. Carusotto, R. Houdré, E. Giacobino, and A. Bramati, Superfluidity of polaritons in semiconductor microcavities, Nat. Phys. 5, 805 (2009).

[12] D. Sanvitto, F. M. Marchetti, M. H. Szymańska, G. Tosi, M. Baudisch, F. P. Laussy, D. N. Krizhanovskii, M. S. Skolnick, L. Marrucci, A. Lemaître, J. Bloch, C. Tejedor, and L. Viña, Persistent currents and quantized vortices in a polariton superfluid, Nat. Phys. 6, 527 (2010).

[13] D. V. Skryabin, D. N. Krizhanovskii, M. S. Skolnick, E. A. Cerda-Méndez, and R. Hartley, Solitons in semiconductor microcavities, Nat. Photon. 6, 204 (2012).

[14] N. Takemura, S. Trebaol, M. Wouters, M. T. Portella-Oberli, and B. Deveaud, Polaritonic Feshbach resonances, Nat. Phys. 10, 500 (2014).

[15] Y.-S. Lee, T. B. Norris, M. Kira, F. Jahnke, S. W. Koch, G. Khitrova, and H. M. Gibbs, Quantum correlations and intraband coherences in semiconductor cavity QED, Phys. Rev. Lett. 83, 5338 (1999).

[16] M. Koch, J. Shah, and T. Meier, Coupled absorber-cavity system: Observation of a characteristic nonlinear response, Phys. Rev. B 57, R2049 (1998).

[17] A. P. D. Love, D. N. Krizhanovskii, D. M. Whittaker, R. Bouchekioua, D. Sanvitto, S. A. Rizeiqi, R. Bradley, M. S. Skolnick, P. R. Eastham, R. André, and L. S. Dang, Intrinsic decoherence mechanisms in the microcavity polariton condensate, Phys. Rev. Lett. 101, 067404 (2008).

[18] V. Kohnle, Y. Léger, M. Wouters, M. Richard, M. T. PortellaOberli, and B. Deveaud, Four-wave mixing excitations in a dissipative polariton quantum fluid, Phys. Rev. B 86, 064508 (2012).

[19] S. Kundermann, M. Saba, C. Ciuti, T. Guillet, U. Oesterle, J. L. Staehli, and B. Deveaud, Coherent control of polariton parametric scattering in semiconductor microcavities, Phys. Rev. Lett. 91, 107402 (2003).

[20] V. Ardizzone, P. Lewandowski, M. H. Luk, Y. C. Tse, N. H. Kwong, A. Lücke, M. Abbarchi, E. Baudin, E. Galopin, J. Bloch, A. Lemaitre, P. T. Leung, P. Roussignol, R. Binder, J. Tignon, and S. Schumacher, Formation and control of Turing patterns in a coherent quantum fluid, Sci. Rep. 3, 3016 (2013).

[21] P. Borri, W. Langbein, U. Woggon, A. Esser, J. R. Jensen, and J. M. Hvam, Biexcitons in semiconductor microcavities, Semicond. Sci. Technol. 18, S351 (2003).
[22] M. Saba, F. Quochi, C. Ciuti, U. Oesterle, J. L. Staehli, B. Deveaud, G. Bongiovanni, and A. Mura, Crossover from exciton to biexciton polaritons in semiconductor microcavities, Phys. Rev. Lett. 85, 385 (2000)

[23] X. Li, T. Zhang, C. N. Borca, and S. T. Cundiff, Manybody interactions in semiconductors probed by optical twodimensional Fourier transform spectroscopy, Phys. Rev. Lett. 96, 057406 (2006).

[24] S. T. Cundiff, T. Zhang, A. D. Bristow, D. Karaiskaj, and X. Dai, Optical two-dimensional Fourier transform spectroscopy of semiconductor quantum wells, Acc. Chem. Res. 42, 1423 (2009).

[25] W. Langbein and B. Patton, Heterodyne spectral interferometry for multi dimensional nonlinear spectroscopy of individual quantum systems, Opt. Lett. 31, 1151 (2006).

[26] D. B. Turner and K. A. Nelson, Coherent measurements of highorder electronic correlations in quantum wells, Nature (London) 466, 1089 (2010).

[27] J. Kasprzak, B. Patton, V. Savona, and W. Langbein, Coherent coupling between distant excitons revealed by twodimensional nonlinear hyperspectral imaging, Nat. Photon. 5, 57 (2011).

[28] C. R. Hall, J. O. Tollerud, H. M. Quiney, and J. A. Davis, Threedimensional electronic spectroscopy of excitons in asymmetric double quantum wells, New J. Phys. 15, 045028 (2013).

[29] P. Dey, J. Paul, J. Bylsma, S. Deminico, and D. Karaiskaj, Continuously tunable optical multidimensional Fourier-transform spectrometer, Rev. Sci. Instrum. 84, 023107 (2013).

[30] L. Yang, T. Zhang, A. D. Bristow, S. T. Cundiff, and S. Mukamel, Isolating excitonic Raman coherence in semiconductors using two-dimensional correlation spectroscopy, J. Chem. Phys. 129, 234711 (2008).

[31] A. D. Bristow, T. Zhang, M. E. Siemens, S. T. Cundiff, and R. P. Mirin, Separating homogeneous and inhomogeneous line widths of heavy-and light-hole excitons in weakly disordered semiconductor quantum wells, J. Phys. Chem. B 115, 5365 (2011).

[32] P. Wen, G. Christmann, J. J. Baumberg, and K. A. Nelson, Influence of multi-exciton correlations on nonlinear polariton dynamics in semiconductor microcavities, New J. Phys. 15, 025005 (2013).

[33] F. Albert, K. Sivalertporn, J. Kasprzak, M. Strauß, C. Schneider, S. Höfling, M. Kamp, A. Forchel, S. Reitzenstein, E. A. Muljarov, and W. Langbein, Microcavity controlled coupling of exciton qubits, Nat. Commun. 4, 1747 (2013).

[34] A. D. Bristow, D. Karaiskaj, X. Dai, T. Zhang, C. Carlsson, K. R. Hagen, R. Jimenez, and S. T. Cundiff, A versatile ultrastable platform for optical multidimensional Fourier-transform spectroscopy, Rev. Sci. Instrum. 80, 073108 (2009).

[35] D. M. Whittaker, P. Kinsler, T. A. Fisher, M. S. Skolnick, A. Armitage, A. M. Afshar, M. D. Sturge, J. S. Roberts, G. Hill, and M. A. Pate, Motional narrowing in semiconductor microcavities, Phys. Rev. Lett. 77, 4792 (1996).

[36] A. D. Bristow, D. Karaiskaj, X. Dai, and S. T. Cundiff, All-optical retrieval of the global phase for two-dimensional Fourier-transform spectroscopy, Opt. Express 16, 18017 (2008).

[37] M. E. Siemens, G. Moody, H. Li, A. D. Bristow, and S. T. Cundiff, Resonance lineshapes in two-dimensional Fourier transform spectroscopy, Opt. Express 18, 17699 (2010). 
[38] C. Ell, J. Prineas, T. R. Nelson Jr., S. Park, H. M. Gibbs, G. Khitrova, S. W. Koch, and R. Houdré, Influence of structural disorder and light coupling on the excitonic response of semiconductor microcavities, Phys. Rev. Lett. 80, 4795 (1998).

[39] A. D. Bristow, D. Karaiskaj, X. Dai, R. P. Mirin, and S. T. Cundiff, Polarization dependence of semiconductor exciton and biexciton contributions to phase-resolved optical two-dimensional Fourier-transform spectra, Phys. Rev. B 79, 161305(R) (2009).

[40] W. Langbein, J. M. Hvam, M. Umlauff, H. Kalt, B. Jobst, and D. Hommel, Binding-energy distribution and dephasing of localized biexcitons, Phys. Rev. B 55, R7383(R) (1997).
[41] G. Moody, R. Singh, H. Li, I. A. Akimov, M. Bayer, D. Reuter, A. D. Wieck, A. S. Bracker, D. Gammon, and S. T. Cundiff, Influence of confinement on biexciton binding in semiconductor quantum dot ensembles measured with two-dimensional spectroscopy, Phys. Rev. B 87, 041304(R) (2013).

[42] G. Khitrova, H. M. Gibbs, M. Kira, S. W. Koch, and A. Scherer, Vacuum Rabi splitting in semiconductors, Nat. Phys. 2, 81 (2006).

[43] E. Giacobino, J.-P. Karr, G. Messin, H. Eleuch, and A. Baas, Quantum optical effects in semiconductor microcavities, C. R. Phys. 3, 41 (2002). 